\title{
Advances in full field microscopy with table-top soft $x$-ray lasers
}

\author{
Carmen S. Menoni*ab Fernando Brizuela $^{\mathrm{ab}}$, Yong Wang ${ }^{\mathrm{ab}}$, Courtney A. Brewer ${ }^{\mathrm{ab}}$, Bradley M. \\ Luther $^{\mathrm{ab}}$, Francesco Pedacia ${ }^{\mathrm{ab}}$, Przemeslaw W. Wachulak ${ }^{\mathrm{ab}}$, Mario C. Marconi ${ }^{\mathrm{ab}}$, Jorge J. Rocca ${ }^{\mathrm{ab}}$, \\ Weilun Chao ac , Erik H. Anderson ${ }^{\text {ac }}$, Yanwei Liu ${ }^{\text {ac }}$, Kenneth A. Goldberg ${ }^{c}$, David T. Attwood ${ }^{\text {acd }}$, \\ Alexander V. Vinogradov ${ }^{e}$, Igor. A. Artyukov ${ }^{e}$, Yuri P. Pershyn ${ }^{f}$, Viktor Kondratenko ${ }^{f}$ \\ ${ }^{a}$ NSF Engineering Research Center for Extreme Ultraviolet Science and Technology \\ ${ }^{\mathrm{b}}$ Colorado State University, Fort Collins, CO, USA 80526 \\ ${ }^{c}$ Lawrence Berkeley National Laboratory, Berkeley, CA, USA 94720 \\ ${ }^{\mathrm{d}}$ University of California, Berkeley, CA, USA 94720 \\ ${ }^{e}$ P.N. Lebedev Physical Institute, Moscow, Russian Federation \\ 'National Technical University 'Kh PI', Kharkov, Ukraine
}

\begin{abstract}
We describe recent advances in the demonstration of table-top full field microscopes that use soft x-ray lasers for illumination. We have achieved wavelength resolution and single shot exposure operation with a very compact $46.9 \mathrm{~nm}$ microscope based on a desk-top size capillary discharge laser. This $\lambda=46.9 \mathrm{~nm}$ microscope has been used to captured full field images of a variety of nanostructure systems and surfaces. In a separate development we have demonstrated a zone plate microscope that uses $\lambda=13.2 \mathrm{~nm}$ laser illumination to image absorption defects in a extreme ultraviolet lithography (EUVL) mask in the same geometry used in a $4 \times$ demagnification EUVL stepper. Characterization of the microscope's transfer function shows it can resolve $55 \mathrm{~nm}$ half period patterns. With these capabilities, the $\lambda=13.2 \mathrm{~nm}$ microscope is well suited for evaluation of pattern and defect printability of EUVL masks for the $22 \mathrm{~nm}$ node.
\end{abstract}

Keywords: soft x-rays, microscopy, extreme ultraviolet lithography, defect inspection, nanoscale imaging

\section{INTRODUCTION}

The development of coherent and incoherent table-top sources of extreme ultraviolet (EUV) and soft x-ray (SXR) light has stimulated the implementation of full field microscopes which owing to the short wavelength illumination provide a spatial resolution significantly better than that of visible microscopes. [1-10]. In this paper we summarize recent advances in the SXR microscopy enabled by table-top soft $x$-ray lasers developed at Colorado State University [11, 12]. Equipped with diffractive and reflective optics, these full field microscopes have been used to image nanostructures, surfaces and absorption patterns in extreme ultraviolet masks with a spatial resolution better than $50 \mathrm{~nm}$ and exposures ranging from $20 \mathrm{sec}$ down to a single laser shot.

\section{SINGLE SHOT -WAVELENGTH RESOLUTION IMAGING WITH A COMPACT SXR MICROSCOPE}

We have built a compact microscope that combines the output from a $46.9 \mathrm{~nm}$ wavelength desk-top Ne-like Ar laser [11]with high quality reflective and diffractive optics allowing transmission and reflection mode imaging with $50 \mathrm{~nm}$ spatial resolution. $[6,7]$ The microscope, including the laser source has a very small foot-print, occupying a portion of a $8 \times 6 \mathrm{sq} \mathrm{ft}$ optical table. A picture of the system is shown in Fig. 1 . 

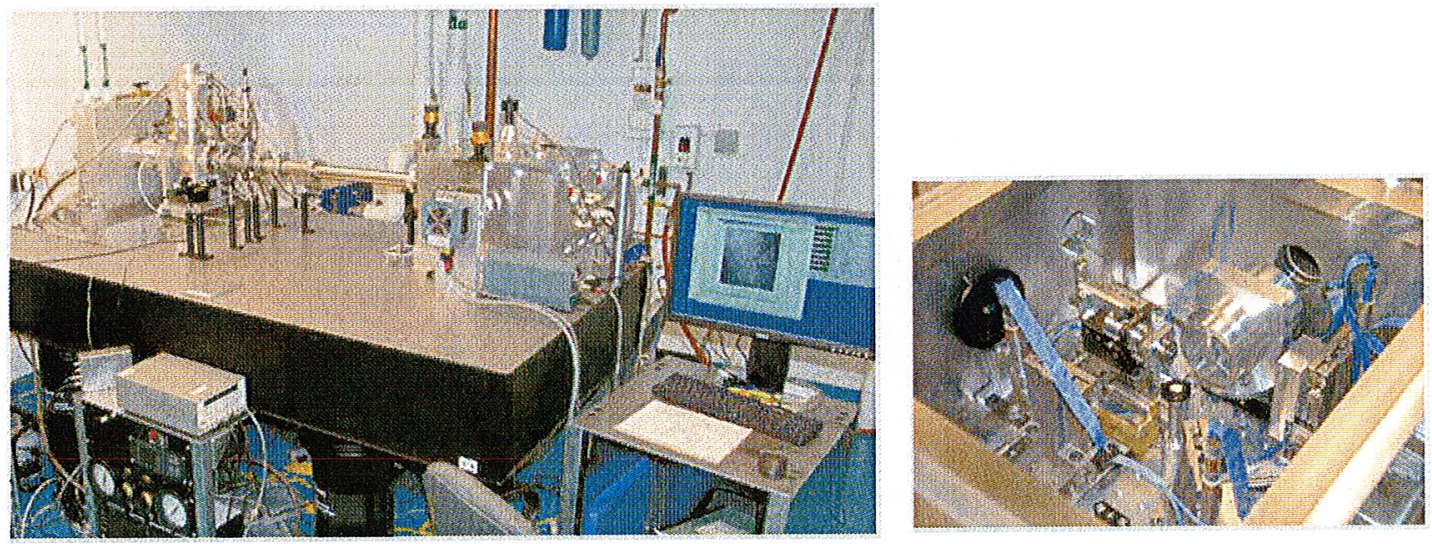

Fig. 1. Left) Picture of the SXR microscope. The laser unit on the left is connected through standard vacuum fittings to the microscope chamber on the right. The entire system fits on a small optical table. Right) Picture of the inside of the microscope chamber.

This microscope uses a Sc/Si coated Schwarzschild condenser to illuminate the object and free standing Fresnel zone plate objectives. Three objectives with $0.12,0.20$, and $0.32 \mathrm{NA}$ are available for this microscope. [13]

The resolving power for each objective zone plate was evaluated constructing modulation transfer curves shown in Fig. 2. The data were collected from the modulation of intensity cross-sections of single shot images of transmission gratings with half-periods varying from $54 \mathrm{~nm}$ to $300 \mathrm{~nm}$. For each objective zone plate, images of the different gratings were obtained and the intensity modulation calculated. Figure 2 shows that the resolution of the microscope increases with increased numerical aperture (NA) of the objective. The highest spatial resolution obtained with the 0.32 NA objective is $\sim 50 \mathrm{~nm}$. These are the first results reported on a SXR microscope that achieves wavelength resolution.

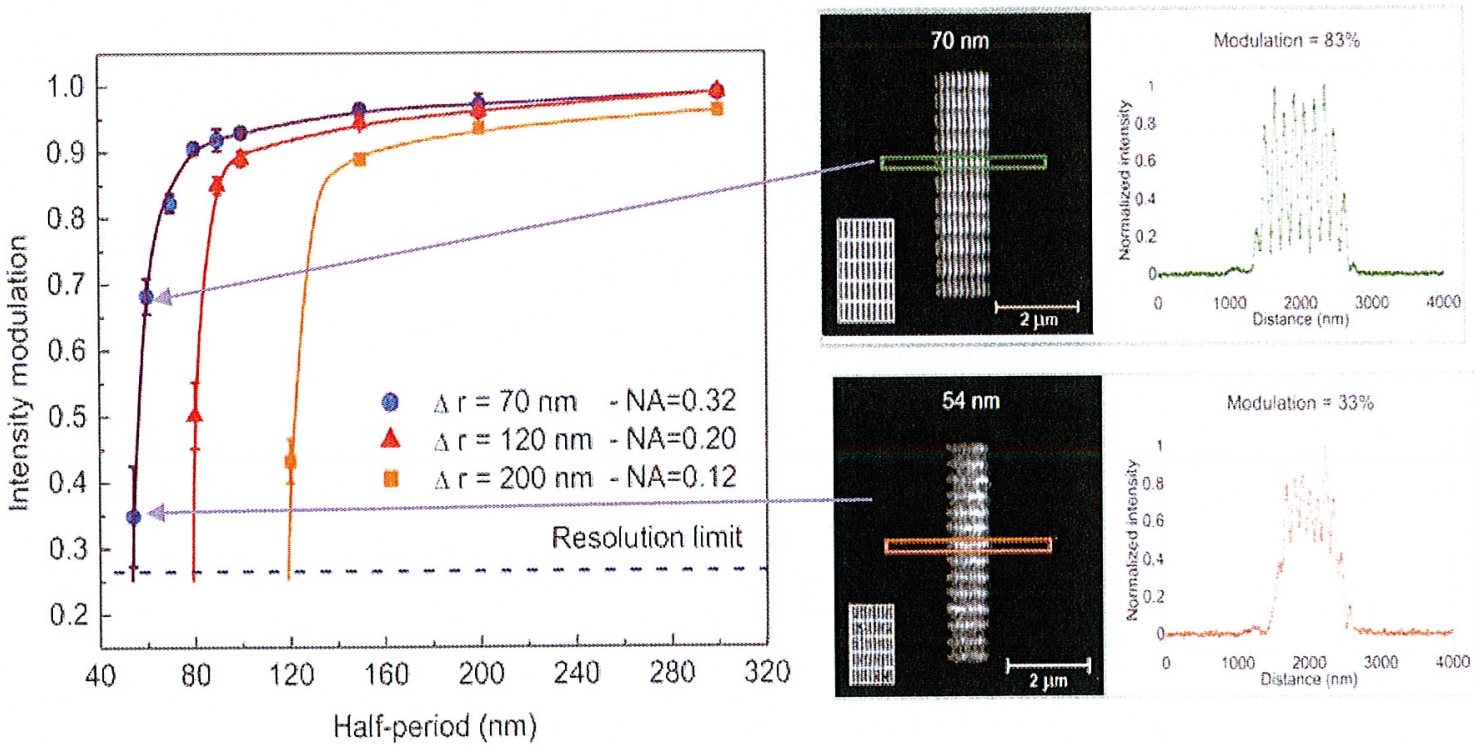

Fig. 2. Measured Modulation Transfer Functions (MTFs) for three objective zone plates. With a 0.32 NA zone plate, a spatial resolution of $50 \mathrm{~nm}$ can be achieved.

Several samples were prepared and imaged with the $\lambda=46.9 \mathrm{~nm}$ microscope to illustrate its capabilities. For transmission imaging sample preparation consisted of laying the objects onto a $\sim 100 \mathrm{~nm}$ thick Si window fabricated on a $5 \times 5 \mathrm{~mm}^{2}$ Si wafer. At $\lambda=46.9 \mathrm{~nm}$, the Si window transmitted $\sim 30 \%$ of the light. A transmission SXR image of a single diatom 
obtained using the $0.32 \mathrm{NA}$ objective with an exposure of 5 seconds is shown in Fig. 3. The fine structure of the diatom is well resolved.

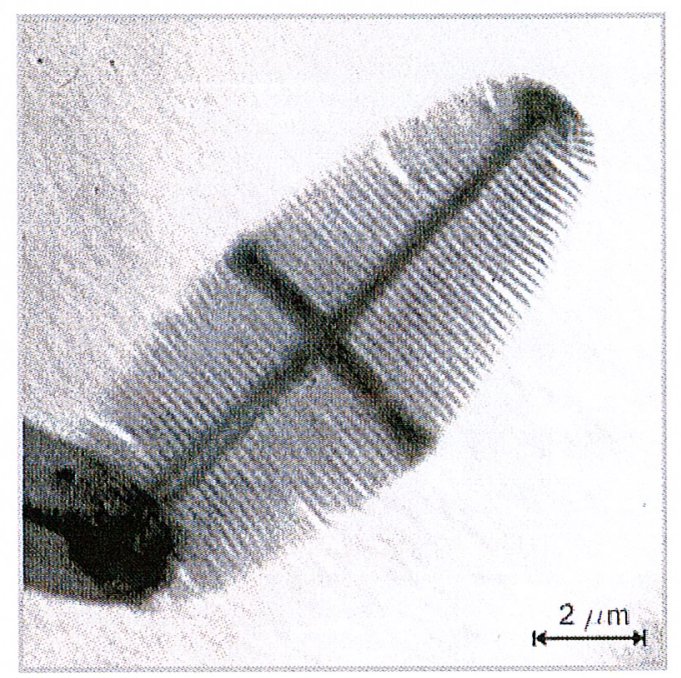

Fig. 3. SXR image of a single diatom obtained with a 5 second exposure using the 0.32 NA objective.

The microscope has also been used to image carbon nanotubes with a diameter of $\sim 50 \mathrm{~nm}$. Fig. 4.a) is a SXR image of a single nanotube obtained with one laser shot. Figure 4.b) shows the same tube as imaged by a scanning electron microscope (SEM) for comparison. [7]

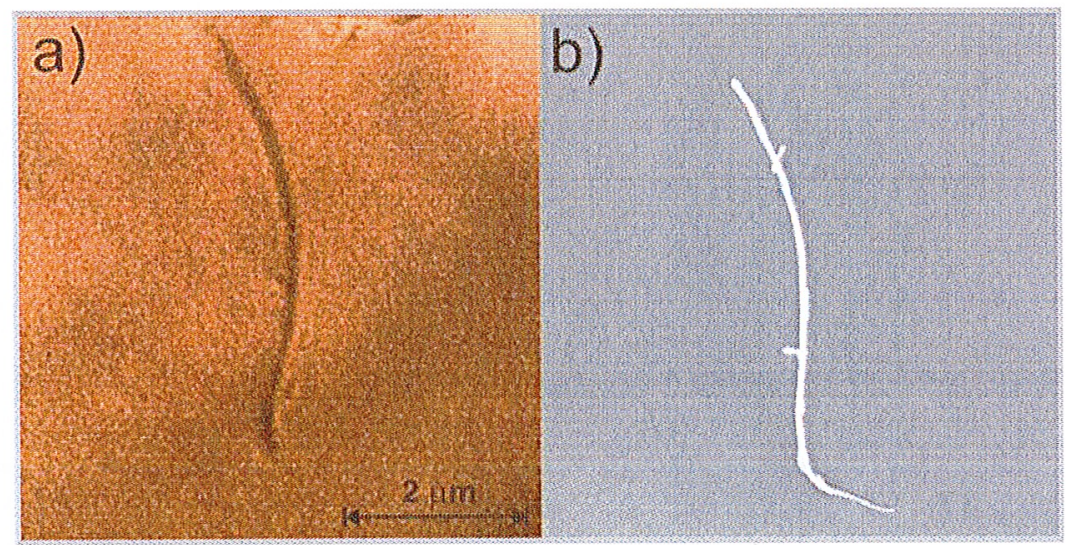

Fig. 4. a) Single shot SXR image of a $\sim 50 \mathrm{~nm}$ wide carbon nanotube on a Si membrane. The image was obtained with a $0.32 \mathrm{NA}$ zone plate. b) Same carbon nanotube as imaged by SEM.

The microscope also operates in reflection configuration. This is accomplished by positioning the sample at $45^{\circ}$ with respect to the direction of the illumination. [6] Because the objective is also at $45^{\circ}$ with respect to the sample, its depth of focus limits to $\sim 2 \mu \mathrm{m}$ the field of view where the image is in focus. Nevertheless, very good quality images with short exposures $(<20 \mathrm{sec})$ can be obtained. This is significant because the reflectivity of most materials is low $(<5 \%)$ at $\lambda=46.9 \mathrm{~nm}$. Figure 5 shows an SXR image of a GaAs nanowire bridging two Al contacts grown on a Si wafer was imaged. The image was obtained with the $0.20 \mathrm{NA}$ objective and an exposure time of 5 seconds corresponding to 15 laser shots. 


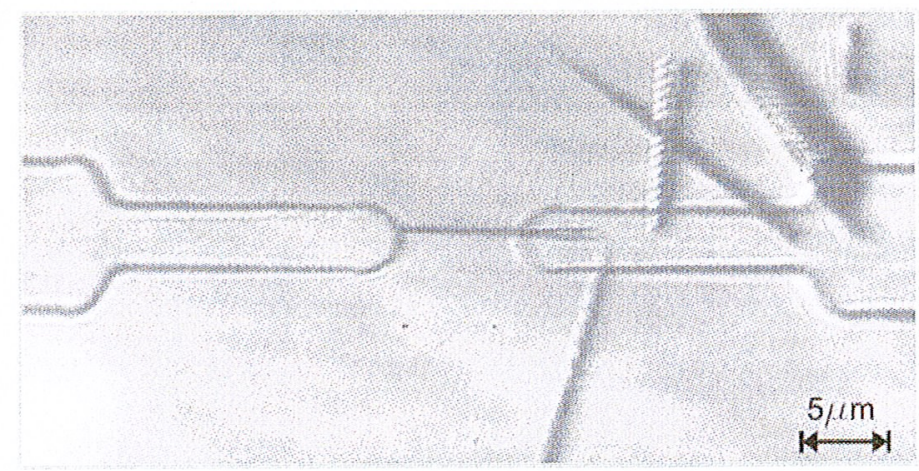

Fig. 5. Reflection image of a GaAs nanowire between two Al contacts on a Si wafer. The SXR image was acquired with a 0.20 NA objective zone plate. In the image, other nanowires that did not successfully bridge the contacts can be seen.

The small penetration depth of the $\lambda=46.9 \mathrm{~nm}$ light combined with the high resolution of the microscope are ideal for the study of surface phenomena in materials. Fig. 6 shows the SXR imaged of the Zr surface obtained with the 0.20 NA objective. In the image grain boundaries and defects are clearly observed.

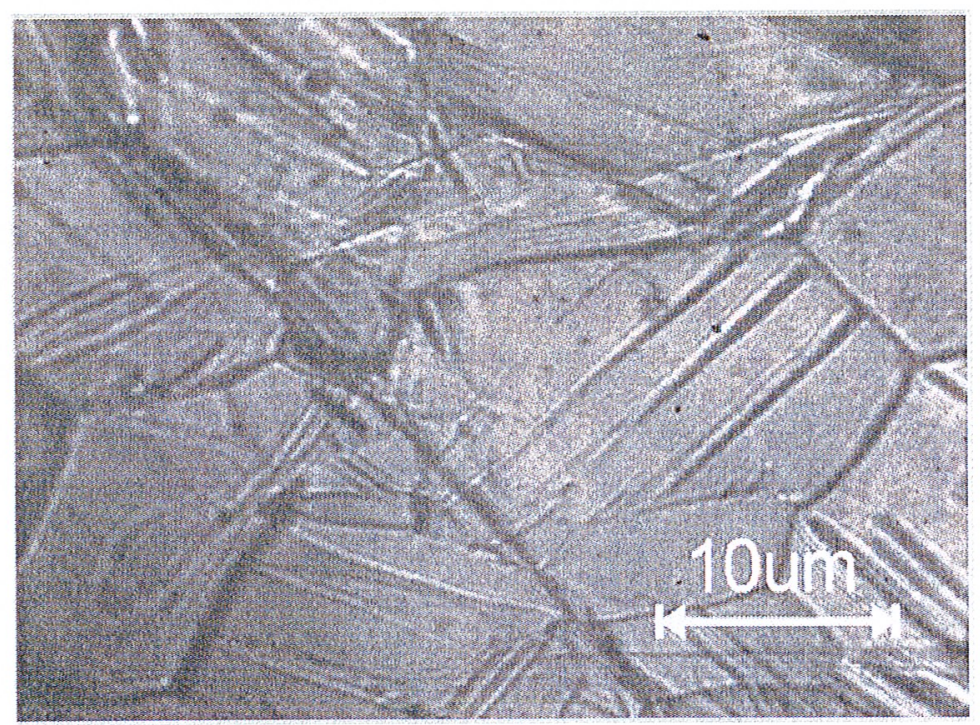

Fig. 6. Reflection mode image of the surface of a strained $\mathrm{Zr}$ sample. The image was obtained with a 0.20 NA objective and an exposure time of 5 seconds.

\section{13.2 NM WAVELENGTH ACTINIC INSPECTION MICROSCOPE}

We demonstrated in 2006 transmission imaging at $\lambda=13.2 \mathrm{~nm}$ with a spatial resolution better than $38 \mathrm{~nm}$, using a zone plate microscope illuminated by a table-top Ni-like Cd laser. [4] With the specific goal of imaging and characterizing defects on Mo/Si reflective EUVL masks, we have recently demonstrated the challenging reflection configuration using a table-top zone plate microscope operating at at $\lambda=13.2 \mathrm{~nm}$. [8]

The $\lambda=13.2 \mathrm{~nm}$ reflection microscope is designed to mimic the imaging conditions of a $4 \times$ demagnification, $0.25 \mathrm{NA}$, EUVL stepper. This was accomplished by setting the angle of the illumination at 6 degrees with respect to the normal 
to the mask surface, by choosing the numerical aperture of the objective to be one forth the NA of the EUVL stepper and by matching the numerical apertures of the condenser to that of the objective. In this way, any printable defect on a EUVL mask is imaged under the same illumination conditions as it would print. This design follows that of an actinic microscope demonstrated by some of us that uses the Advanced Light Source for illumination. [14] Figure 7 shows a schematic diagram of the setup along with a picture of the microscope chamber where the beam path has been marked. The $\lambda=13.2 \mathrm{~nm}$ laser output is guided by a Mo/Si mirror to the condenser that illuminates the test object. The condenser with $0.065 \mathrm{NA}$ was fabricated by electron beam lithography on a Si3N4 membrane. The structure also contains an aperture that clears the path for the zone plate objective to form the image on the CCD detector at normal incidence to the mask surface. An off-axis design was used for the objective zone plate. In this design illustrated in Fig. 8, an offaxis pupil is overlapped onto a 'parent' zone plate, limiting the numerical aperture of the lens. Because the zones within the pupil are concentric to the axis of the 'parent' zone plate, the image is projected onto a plane perpendicular to the lens. Due to the small angle of incidence of the incoming light, a region opposite to the lens is left uncoated, creating a thin $\mathrm{Si}_{3} \mathrm{~N}_{4}$ window that allows ample throughput of the $13.2 \mathrm{~nm}$ light. The insert in Fig. 8 shows the outer zone widths of the zone plate that are $40 \mathrm{~nm}$ wide. The effective NA of the objective equals $0.065 \mathrm{NA}$ matching that of the condenser.
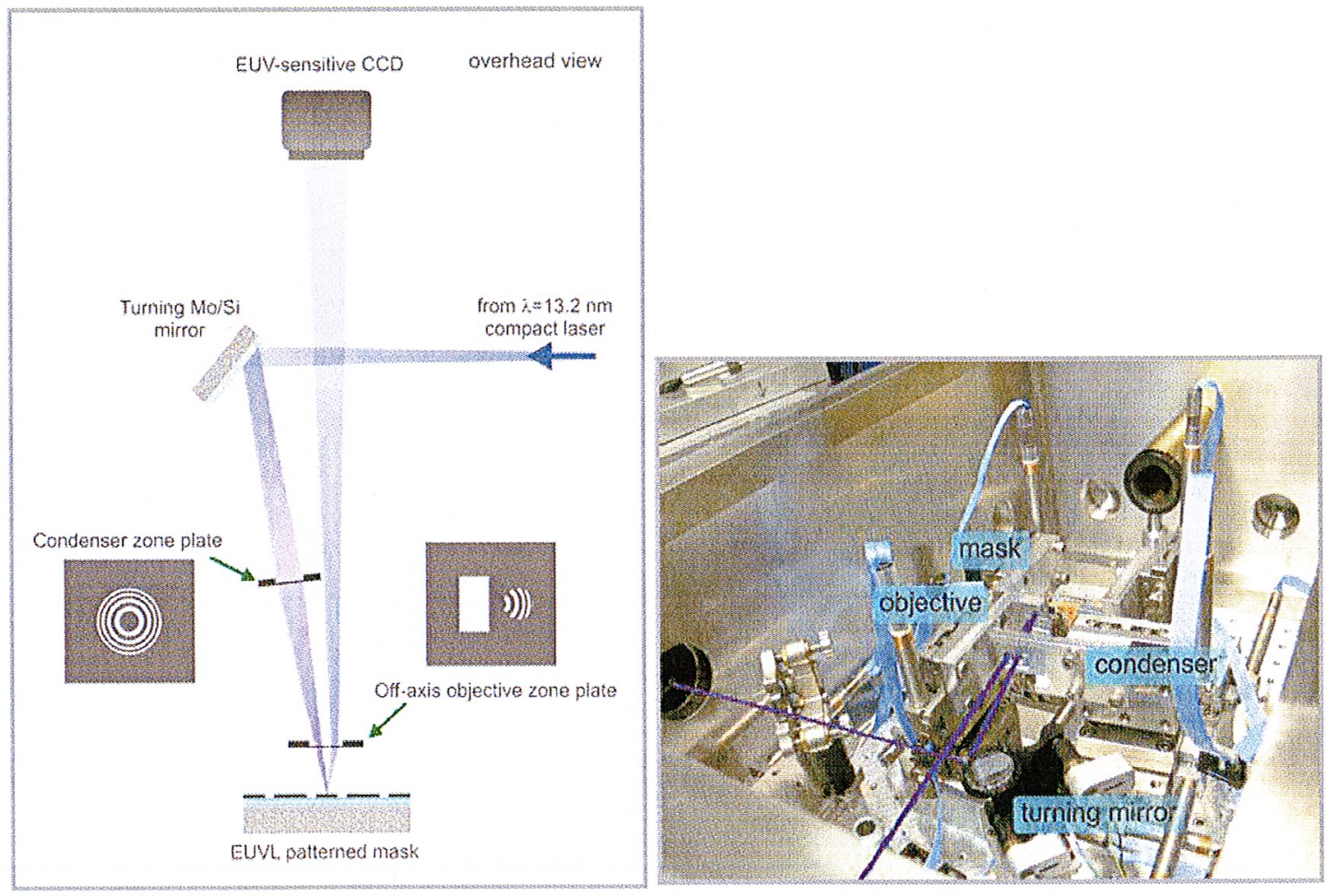

Fig. 7. a) Schematic representation of the $13.2 \mathrm{~nm}$ wavelength microscope for EUVL mask inspection. b) Photograph of the microscope chamber. The beam enters the chamber from the port on the left of the image. The line was added to indicate the beam path.

A EUVL mask simile consisting of a Mo/Si stack with $\sim 63 \%$ reflectance at $\lambda=13.2 \mathrm{~nm}$ containing absorption features consisting of elbow patterns with half-pitch lines ranging from 80 to $200 \mathrm{~nm}$ was fabricated and used to test the microscope. Figure 9 shows SXR reflection images of (a) $80 \mathrm{~nm}$, (b) $100 \mathrm{~nm}$, (c) $120 \mathrm{~nm}$, and (d) $140 \mathrm{~nm}$ half-pitch structures with their corresponding intensity cross-sections. The images have a field-of-view of $25 \mu \mathrm{m}^{2}$ and were obtained with a 20 second exposure time. The high modulation values indicate that even the $80 \mathrm{~nm}$ features are fully resolved. The fact that the vertical and horizontal lines can be resolved at the same time indicates that the astigmatism of the system is low. 


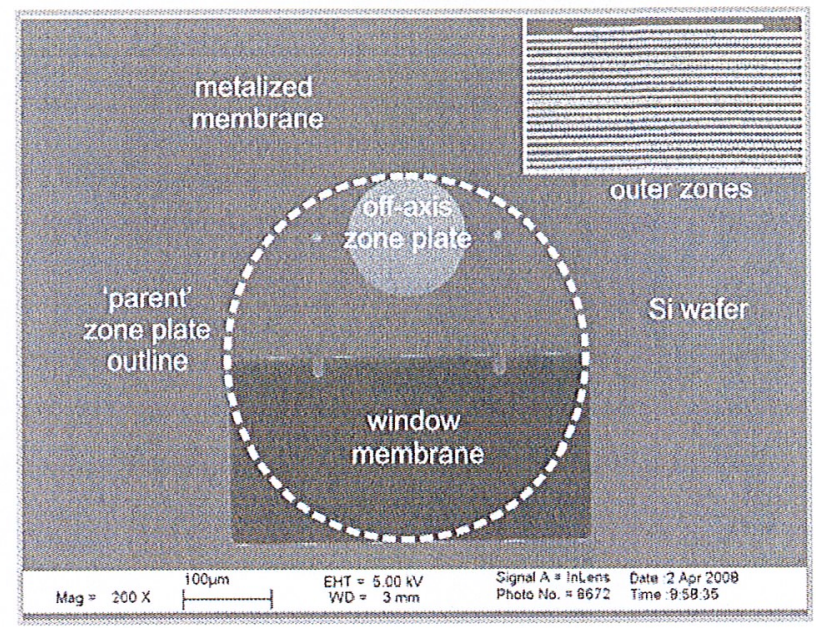

Fig. 8. SEM image of the off-axis objective zone plate. The insert in the top-right corner of the image shows a magnified image of the $40 \mathrm{~nm}$ outer zones of the zone plate. The effective NA of the off-axis objective is 0.0625 .
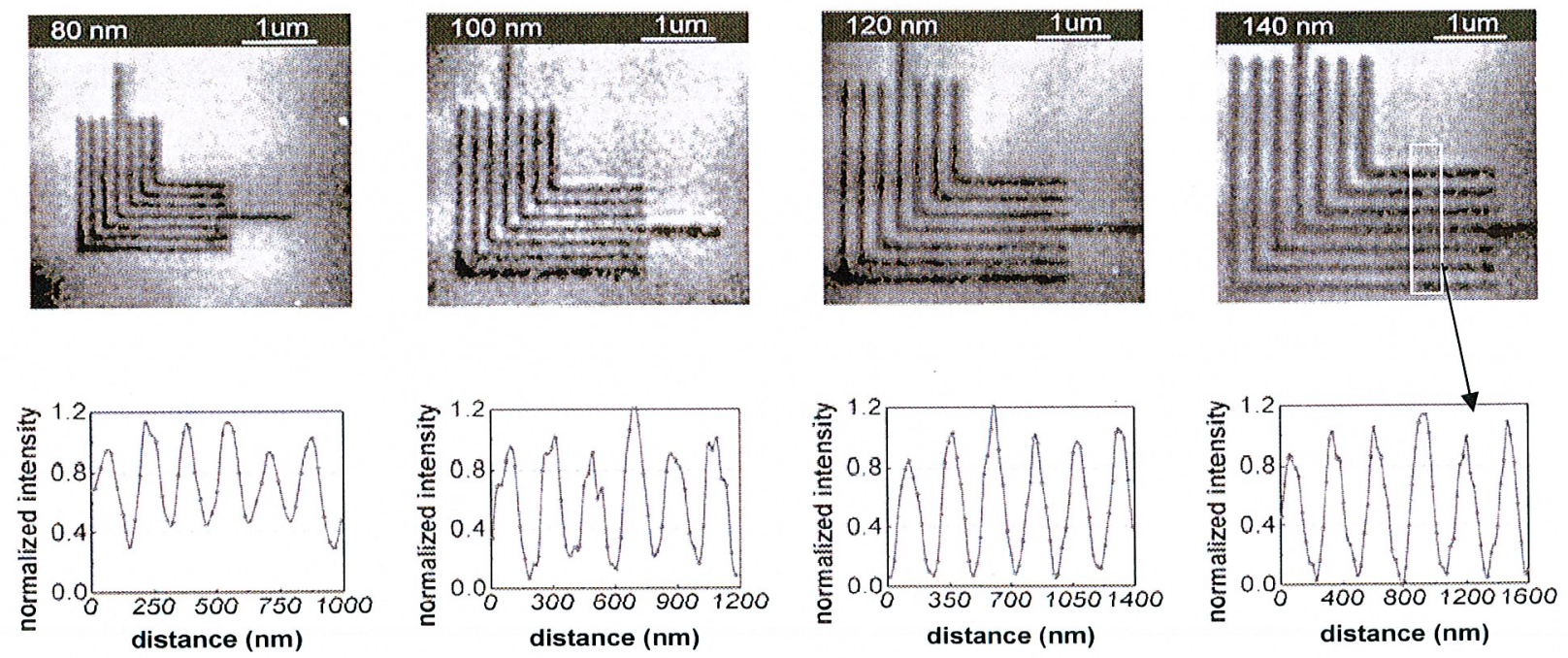

Fig. 9. SXR images and corresponding intensity cross-sections of elbow patterns of (a) $80 \mathrm{~nm}$, (b) $100 \mathrm{~nm}$, (c) $120 \mathrm{~nm}$, and (d) 140 $\mathrm{nm}$ half-pitch printed on a Mo/Si multilayer coated mirror. The images were obtained with 5 seconds exposure times. The high modulation of the cross-sections indicate that the features are fully resolved.

Since no smaller grating structures were available at the time of the experiments, the resolution limit of the microscope corresponding to a modulation of $26.5 \%$ was evaluated by measuring the $10-90 \%$ variation in the intensity in the image of a sharp edge. This analysis yielded a value of $110 \mathrm{~nm}$ which was also independently obtained through an image analysis method previously described. [15]To construct the modulation transfer function of the $\lambda=13.2 \mathrm{~nm}$ reflection microscope, the results of the grating test and half the value of the knife-edge analysis were combined. This is possible because the illumination conditions of this microscope are practically incoherent as a result of the low spatial coherence of the illumination and the selection of the same objective NA and the condenser NA. These results are plotted in Fig. 
10 show the microscope has achieved a spatial resolution, $55 \mathrm{~nm}$, that exceeds the specifications set for the $22 \mathrm{~nm}$ technology half-pitch node.

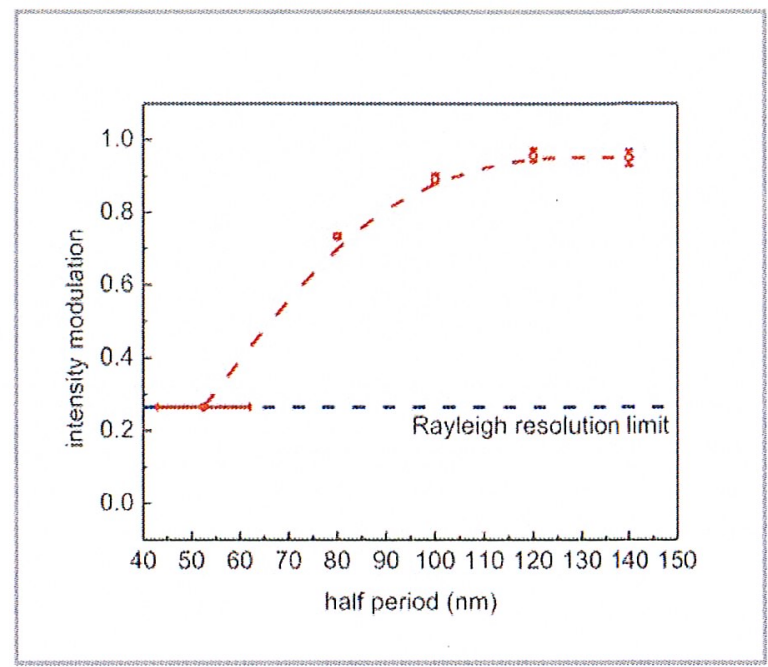

Fig. 10. Measured Modulation Transfer Function (MTF) of the reflection mode microscope. The resolution of the imaging system is $\sim 55 \mathrm{~nm}$.

This first demonstration of a table-top full-field microscope for actinic characterization of EUVL masks opens the path for on-site mask inspection and will contribute to the development of EUV Lithography as a viable technique for the new generation of semiconductor chips. Forecasted improvements in the laser output power are expected to reduce the exposure time to a few seconds.

\section{SUMMARY}

High average power table-top SXR lasers at Colorado State University have enabled the implementation of compact wavelength scalable microscopes capable to image nanostructures and surfaces with $\sim 50 \mathrm{~nm}$ spatial resolution. We have demonstrated a practical microscope operating at $\lambda=46.9 \mathrm{~nm}$ that captures full field images with wavelength resolution and with a single laser shot. This capability will enable tracking of dynamic phenomena in nanoscale systems. We have also implemented a table-top zone plate microscope at $\lambda=13.2 \mathrm{~nm}$ that using a geometry similar to a EUVL stepper images and characterizes absorption defects in EUVL masks with $55 \mathrm{~nm}$ resolution. This is a first demonstration towards the implementation of a practical defect inspection microscope. Progress in SXR lasers towards increased output power and shorter wavelengths will generate new opportunities for table-top short wavelength microscopy to support nanoscience and nanotechnology.

\section{ACKNOWLEDGMENTS}

We acknowledge the contribution of Dr. Georgiy Vaschenko and the support of the Engineering Research Centers Program of the National Science Foundation under NSF Award Number EEC-0310717. 


\section{REFERENCES}

1. D.S. DiCicco, D. Kim, R. Rosser, And S. Suckewer, Ist Stage In The Development Of A Soft-X-Ray Reflection Imaging Microscope In The Schwarzschild Configuration Using A Soft-X-Ray Laser At $18.2 \mathrm{~nm}$. Optics Letters, 1992. 17(2): p. 157-159.

2. L.B. DaSilva, J.E. Trebes, R. Balhorn, S. Mrowka, E. Anderson, D.T. Attwood, T.W. Barbee, J. Brase, M. Corzett, J. Gray, J.A. Koch, C. Lee, D. Kern, R.A. London, B.J. Macgowan, D.L. Matthews, And G. Stone, X-Ray Laser Microscopy Of Rat Sperm Nuclei. Science, 1992. 258(5080): p. 269-271.

3. W.L. Chao, B.D. Harteneck, J.A. Liddle, E.H. Anderson, And D.T. Attwood, Soft X-Ray Microscopy At A Spatial Resolution Better Than 15nm. Nature, 2005. 435(7046): p. 1210-1213.

4. G. Vaschenko, C. Brewer, F. Brizuela, Y. Wang, M.A. Larotonda, B.M. Luther, M.C. Marconi, J.J. Rocca, And C.S. Menoni, Sub-38 nm Resolution Tabletop Microscopy With $13 \mathrm{~nm}$ Wavelength Laser Light. Optics Letters, 2006. 31(9): p. 1214-1216.

5. G. Vaschenko, F. Brizuela, C. Brewer, M. Grisham, H. Mancini, C.S. Menoni, M.C. Marconi, J.J. Rocca, W. Chao, J.A. Liddle, E.H. Anderson, D.T. Attwood, A.V. Vinogradov, I.A. Artioukov, Y.P. Pershyn, And V.V. Kondratenko, Nanoimaging With A Compact Extreme-Ultraviolet Laser. Optics Letters, 2005. 30(16): p. 20952097.

6. F. Brizuela, G. Vaschenko, C. Brewer, M. Grisham, C.S. Menoni, M.C. Marconi, J.J. Rocca, W. Chao, J.A. Liddle, E.H. Anderson, D.T. Attwood, A.V. Vinogradov, I.A. Artioukov, Y.P. Pershyn, And V.V. Kondratenko, Reflection Mode Imaging With Nanoscale Resolution Using A Compact Extreme Ultraviolet Laser. Optics Express, 2005. 13(11): p. 3983-3988.

7. C.A. Brewer, F. Brizuela, P. Wachulak, D.H. Martz, W. Chao, E.H. Anderson, D.T. Attwood, A.V. Vinogradov, I.A. Artyukov, A.G. Ponomareko, V.V. Kondratenko, M.C. Marconi, J.J. Rocca, And C.S. Menonil, Single-Shot Extreme Ultraviolet Laser Imaging Of Nanostructures With Wavelength Resolution. Optics Letters, 2008. 33(5): p. 518-520.

8. F. Brizuela, Y. Wang, C.A. Brewer, F. Pedaci, W. Chao, E.H. Anderson, Y. Liu, K.A. Goldberg, P. Naulleau, P. Wachulak, M.C. Marconi, D.T. Attwood, J.J. Rocca, And C.S. Menoni, Microscopy Of Extreme Ultraviolet Lithography Masks With $13.2 \mathrm{~nm}$ Tabletop Laser Illumination. Optics Letters, 2009. 34(3): p. 271-273.

9. P.A.C. Takman, H. Stollberg, G.A. Johansson, A. Holmberg, M. Lindblom, And H.M. Hertz, High-Resolution Compact X-Ray Microscopy. Journal Of Microscopy-Oxford, 2007. 226(2): p. 175-181.

10. J.J. Park, D.S. Kim, S.C. Jeon, J. Park, K.H. Lee, J.H. Lee, K.N. Kim, J.J. Yoo, And C.H. Nam, Soft X-Ray Microscope Constructed With A PMMA Phase-Reversal Zone Plate. Optics Letters, 2009. 34(3): p. 235-237.

11. S. Heinbuch, M. Grisham, D. Martz, And J.J. Rocca, Demonstration Of A Desk-Top Size High Repetition Rate Soft $X$-Ray Laser. Optics Express, 2005. 13(11): p. 4050-4055.

12. J.J. Rocca, Y. Wang, M.A. Larotonda, B.M. Luther, M. Berrill, And D. Alessi, Saturated 13.2 Nm High-RepetitionRate Laser In Nickellike Cadmium (Vol 30, Pg 2581, 2005). Optics Letters, 2006. 31(1): p. 129-129.

13. E.H. Anderson, Specialized Electron Beam Nanolithography For EUV And X-Ray Diffractive Optics. IEEE Journal Of Quantum Electronics, 2006. 42(1-2): p. 27-35.

14. K.A. Goldberg, A. Barty, Y.W. Liu, P. Kearney, Y. Tezuka, T. Terasawa, J.S. Taylor, H.S. Han, And O.R. Wood, Actinic Inspection Of Extreme Ultraviolet Programmed Multilayer Defects And Cross-Comparison Measurements. Journal Of Vacuum Science \& Technology B, 2006. 24 (6): p. 2824-2828.

15. P.W. Wachulak, C.A. Brewer, F. Brizuela, C.S. Menoni, W. Chao, E.H. Anderson, R.A. Bartels, J.J. Rocca, And M.C. Marconi. Analysis Of Extreme Ultraviolet Microscopy Images Of Patterned Nanostructures Based On A Correlation Method. 2008. Journal Of The Optical Society Of America B-Optical Physics 25(7), p. B20-B26. 


\section{SCLAI MER}

This document was prepared as an account of work sponsored by the United States Government. While this document is believed to contain correct information, neither the United States Government nor any agency thereof, nor The Regents of the University of California, nor any of their employees, makes any warranty, express or implied, or assumes any legal responsibility for the accuracy, completeness, or usefulness of any information, apparatus, product, or process disclosed, or represents that its use would not infringe privately owned rights. Reference herein to any specific commercial product, process, or service by its trade name, trademark, manufacturer, or otherwise, does not necessarily constitute or imply its endorsement, recommendation, or favoring by the United States Government or any agency thereof, or The Regents of the University of California. The views and opinions of authors expressed herein do not necessarily state or reflect those of the United States Government or any agency thereof or The Regents of the University of California.

This work was supported by the Director, Office of Science, of the U.S. Department of Energy under Contract No. DE-AC02-05CH11231. 